\title{
Multi-view fast-ion D-alpha spectroscopy diagnostic at ASDEX Upgrade
}

\author{
B. Geiger, ${ }^{\text {a) }}$ R. Dux, R.M. McDermott, S. Potzel, M. Reich, F. Ryter, M. Weiland, D. Wünderlich, and the \\ ASDEX Upgrade team ${ }^{b}$ ) \\ Max-Planck-Institut für Plasmaphysik, EURATOM Association, Boltzmannstr. 2, 85748 Garching, \\ Germany \\ M. Garcia-Munoz \\ Faculty of Physics, University of Seville, Spain
}

(Dated: 31 October 2013)

\begin{abstract}
A novel fast-ion D-alpha (FIDA) diagnostic that is based on charge exchange spectroscopy has been installed at ASDEX Upgrade. The diagnostic uses a newly developed high-photon-throughput spectrometer together with a low-noise EM-CCD camera that allow measurements with $2 \mathrm{~ms}$ exposure time. Absolute intensities are obtained by calibrating the system with an integrating sphere and the wavelength dependence is determined to high accuracy using a neon lamp. Additional perturbative contributions to the spectra, such as $\mathrm{D}_{2}$-molecular lines, the Stark broadened edge D-alpha emission, and passive FIDA radiation have been identified and can be subtracted or avoided experimentally. The FIDA radiation from fast deuterium ions after charge exchange reactions can therefore be analyzed continuously without superimposed line emissions at large Doppler shifts. Radial information on the fast ions is obtained from radially distributed lines of sight. The investigation of the fast-ion velocity distribution is possible due to three different viewing geometries. The independent viewing geometries access distinct parts of the fast-ion velocity space and make tomographic reconstructions possible.
\end{abstract}

PACS numbers: 52.55.Fa, 52.50.Gj, 52.65.Cc, 52.70.Kz, 52.70.Nc

\section{INTRODUCTION}

Good confinement of fast ions generated by neutral beam injection (NBI), ion cyclotron heating or fusion processes is essential in fusion devices because these supra-thermal particles are responsible for plasma heating and current drive and can be critical for the machine safety ${ }^{2}$. Their distribution function must consequently be investigated by diagnostics that can access different parts of the corresponding six dimensional phase space. Several approaches have been developed to monitor the fast ions, such as the measurement of neutrons ${ }^{3}$, gamma rays ${ }^{4}$, Doppler shifted micro waves ${ }^{5}$ and fast-ion $\operatorname{losses}^{6}$. In addition, a widely applied technique to obtain information on the fast ions is based on their charge exchange reactions with neutrals. Along NBI lines, fast ions can capture electrons from the injected neutrals and surrounding halo neutrals with high probability. If the fast ions are neutralized by these reactions, they are no longer confined by the magnetic field and can be detected by neutral particle analyzers (NPA) ${ }^{7}$, located outside the plasma. In addition, their line radiation due to the bound electrons can be analyzed by means of spectroscopy. The charge exchange radiation from fast helium ions has, for example, been analyzed at $\mathrm{TFTR}^{8}$ to obtain information on the alpha particle population in D-T plasmas. In recent years, however, the analysis of fast deuterium

\footnotetext{
a) Electronic mail: benedikt.geiger@ipp.mpg.de

b) For authors list, see U. Stroth et. al., Nucl. Fusion 53, 104003A (2013)
}

ions, i.e. the FIDA method ${ }^{9}$, has become a valuable tool at several fusion devices ${ }^{10-15}$. The FIDA method analyzes the strongly Doppler shifted Balmer alpha radiation $\left(\lambda_{0}=656.1 \mathrm{~nm}, n=3 \rightarrow 2\right)$ and makes use of several lines of sight (LOS) that intersect a given NBI path. Good radial resolution of the measurement is possible as the main part of the FIDA radiation is emitted directly after the charge exchange reactions ${ }^{16}$. Information on fast-ion density profiles can consequently be obtained from the spectral intensities that are measured using radially distributed LOS. Information on different parts of the fast-ion velocity space can be obtained by analyzing different wavelength regions of the FIDA radiation and by using viewing geometries with different angles to the magnetic field. However, it should be noted that the analysis of the FIDA radiation is demanding: Forward modelling of the spectra is needed for a quantitative interpretation and the signal strengths are relatively weak. The FIDA radiation can be comparable in intensity to the bremsstrahlung and other passive components and is in part superimposed by the NBI induced beam and halo emission. Therefore, optimized LOS geometries, spectrometers and cameras are needed to enable a detailed analysis of the fast ion distribution.

In this paper, the FIDA diagnostic at ASDEX Upgrade $^{1}$ is presented. This system was designed and built after the feasibility of the technique was demonstrated using an existing CXRS diagnostic in $2010^{16}$. First, the extended LOS geometries and new spectrometer setup is shown, followed by a short description of the applied calibration techniques. The passive spectral contributions that can be superimposed on the FIDA radiation and thereby limit the measurement capabilities are 

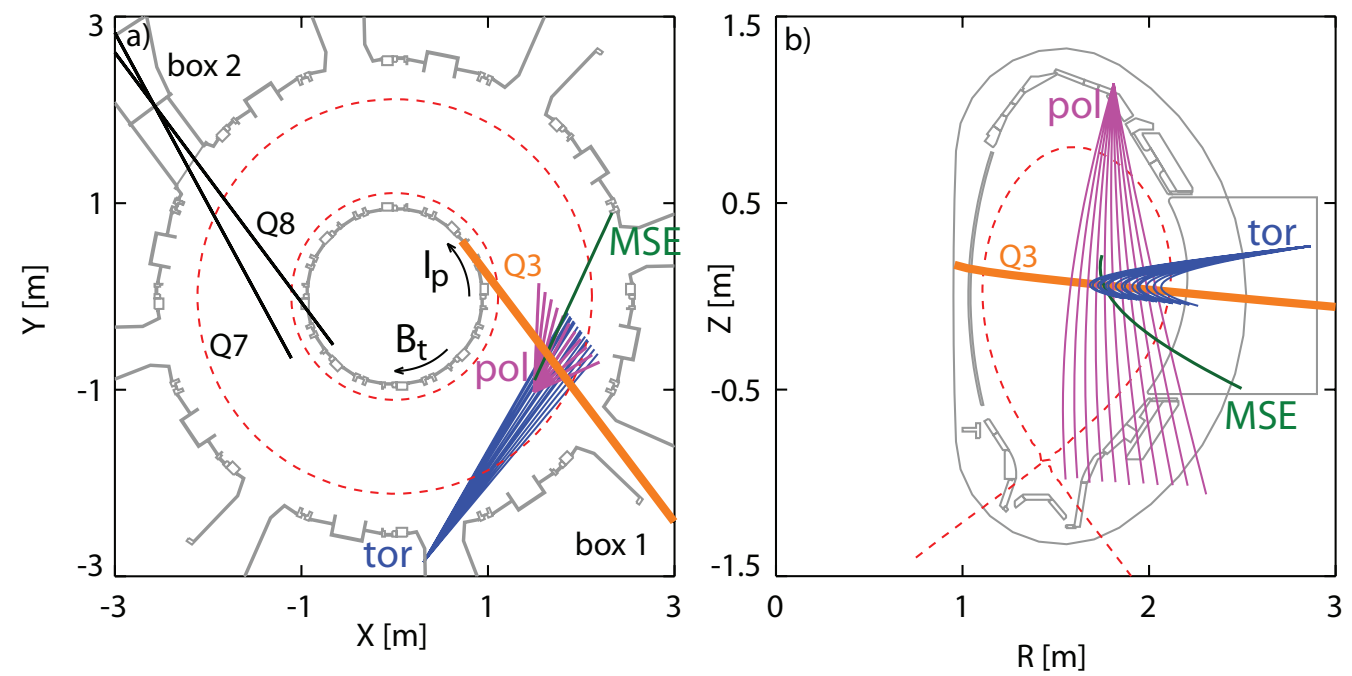

FIG. 1. Top-down (a) and a poloidal view (b) on the LOS-setup of the FIDA diagnostic at ASDEX Upgrade. The toroidal LOS are indicated in blue color and the poloidal ones are shown in purple. The MSE LOS is plotted in green. The NBI source Q3, which is used as a diagnostic beam, is sketched in orange and the separatrix position is illustrated by red, dashed lines.

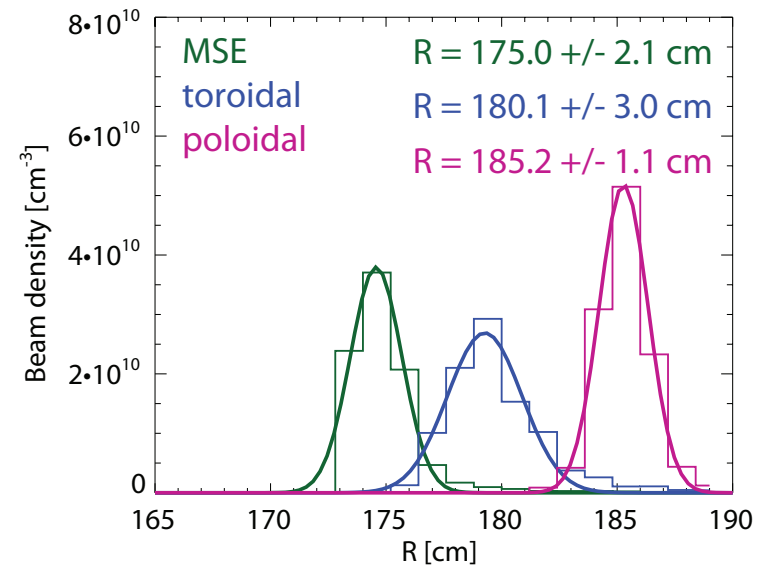

FIG. 2. Radial resolution of three lines of sight of the FIDA diagnostic, based on the calculated neutral density present along the path of NBI Q3.

discussed in the third section. Section four reports on the NBI induced active spectral contributions. The beam, halo and FIDA radiation observed by the three different viewing geometries is presented and the observed regions in the fast-ion velocity space are introduced. Moreover, the possibility to analyze time traces and radial profiles of the FIDA light is presented. Finally, a short summary and outlook are given.

\section{DIAGNOSTIC SETUP}

\section{A. Line of sight setup}

ASDEX Upgrade is a medium size tokamak with major and minor radii of $1.65 \mathrm{~m}$ and $0.5 \mathrm{~m}$ that is equipped with a flexible and powerful set of heating systems. Two NBI boxes, each with four $2.5 \mathrm{MW}$ sources, can generate fast deuterium ions with a full energy of $60 \mathrm{keV}$ and $93 \mathrm{keV}$, respectively. The FIDA diagnostic is focused on one of the $60 \mathrm{keV}$ heating beams of NBI box 1, labeled Q3. As sketched in figure 1, all of the lines of sight of the FIDA diagnostic intersect this beam at different radial positions and consequently allow the analysis of radial profiles. 15 toroidal LOS, plotted in blue, are available that view horizontally from their optical head into the co-current direction. The toroidal LOS thereby form angles with the magnetic field lines from $12^{\circ}$ for the central LOS to $21^{\circ}$ for the LOS close to the plasma edge. As can be seen in figure $1 \mathrm{~b}$, a second optical head is located at the top of the machine. It holds 11 poloidal LOS that are directed downwards on NBI Q3 and form angles to the magnetic field lines between $68^{\circ}$ and $85^{\circ}$ (from center to edge). A third viewing geometry is defined by a single, central LOS, shown in green in figure 1 . This line of sight, which has an angle of about $157^{\circ}$ to the magnetic field lines, was originally installed for motional Stark effect (MSE) measurements ${ }^{17}$ but is now used by the FIDA diagnostic.

The different LOS geometries with respect to the magnetic field lines were chosen because they enable different parts of the fast-ion velocity space to be accessed. In addition, the LOS are arranged such that they yield good radial resolution. The resolution of a given LOS of the FIDA diagnostic is determined by the radial positions at which it intersects the neutral density present along the path of NBI Q3. As shown in figure 2 for three representative LOS, only a limited range of radial positions are encountered. The half widths of the predicted beam and halo neutral density distribution, encountered by the toroidal, poloidal and the MSE LOS are in the range of only $3 \mathrm{~cm}$. This radial resolution is small compared to the minor radius of ASDEX Upgrade and, hence, allows 
to study radial profiles.

\section{B. Spectrometer and camera setup}

The light collected by optics in the torus is transmitted with $400 \mu \mathrm{m}$ thick fibers to a patch panel. From this panel, 15 fibers can be simultaneously connected to one Czerny-Turner-like spectrometer that was designed to obtain a high throughput of photons in combination with good imaging properties and high spectral resolution. The spectrometer uses a holographic grating with 2000 lines $/ \mathrm{mm}$, has an opening angle $\phi$ of $20^{\circ}$, and consists of two Leica objective lenses with focal lengths of $180 \mathrm{~mm}$ and f-numbers of 2.8. As sketched schematically in figure 3, the first lens parallelizes the light emitted by the fibers. The second objective is used to focus the dispersed light of order -1 onto the CCD camera, which is attached to the spectrometer exit. The CCD camera ${ }^{18}$ has a $512 \times 51216 \mu \mathrm{m}$ pixel chip and is optimized to detect relatively low signals with a high dynamic range (16 bit). It is cooled to $-70^{\circ} \mathrm{C}$ by a Peltier element, reducing thermal noise, and uses an electron multiplication (EM) gain of the generated photo electrons to reduce the readout noise. An EM multiplication factor of 15 is typically used and it is operated in the frame-transfer mode, which is based on shifting the generated photo-electrons to a second, non-illuminated part of the CCD-chip before the read out. The readout time of the camera is $2 \mathrm{~ms}$. This is enabled by the $10 \mathrm{MHz} /$ pixel readout rate and by defining 16 regions of interest (ROI) in which the pixels are binned in the vertical direction. The ROIs correspond to one dark channel for the calibration and to the 15 fibers that are vertically stacked at the spectrometer entrance as the grating does not disperse in this direction. In the horizontal direction the 512 pixels per channel monitor the dispersed light in a spectral range of about $14 \mathrm{~nm}$ (at $656 \mathrm{~nm}$ ) with a dispersion per pixel of $0.027 \mathrm{~nm}$. The spectral resolution of the diagnostic is, unfortunately, not represented by this value but is rather $0.16 \mathrm{~nm}$. This is due to the spectrometer's entrance slit which has a width of $100 \mu \mathrm{m}$ and causes a narrow spectral line with at least this size to be measured when imaged onto the CCD.

As shown in figure 3, the spectrometer design allows us to insert a high pass interference filter between the first lens and the grating that blocks the un-shifted and typically intense part of the D-alpha line. The latter can cause scattered radiation in the spectrometer and can significantly disturb the spectra by saturation effects. In particular when strong edge $\mathrm{D}$-alpha radiation is present (usually during plasma operation with electron densities above $2 \times 10^{19} \mathrm{~m}^{-3}$ ) the filter is needed. The filter, however, restricts the analysis of the FIDA radiation to the red-shifted part of the spectrum as it strongly attenuates the light below $657 \mathrm{~nm}$.

\section{Calibration}

A proper calibration of the FIDA diagnostic is needed for a quantitative interpretation of the measurement and for comparisons with modelling results. The wavelength calibration of the diagnostic is performed by measuring the well-known and tabulated ${ }^{19}$ spectrum of neon which has, as shown in figure $4 \mathrm{a}$, two strong emission lines between $655 \mathrm{~nm}$ and $668 \mathrm{~nm}$. These emission lines appear at slightly different horizontal positions in the 16 camera channels because the vertical alignment of the fibers at the spectrometer entrance causes an additional angle between the light rays and the grating. The resulting parabola shaped channel dependence of the centrally measured wavelength, as shown in figure $4 \mathrm{~b}$, must consequently be taken into account when allocating wavelengths to the pixel positions.

The intensity calibration of the FIDA diagnostic is obtained using an integrating sphere ${ }^{20}$ that can be placed in front of the optical heads of the FIDA diagnostic when the vessel of ASDEX Upgrade is open and accessible. The sphere emits spatially uniform light in the visible range with a well-known, continuous spectrum. By measuring the light of the sphere that reaches the FIDA diagnostic, calibration factors from the measured counts/s to actually emitted photons $/\left(\mathrm{s} \mathrm{sr} \mathrm{m}^{2} \mathrm{~nm}\right)$ in the vessel can be determined. However, before applying these calibration factors to the raw data obtained during plasma experiments, an offset and smear effect must be subtracted. The offset is measured before every discharge when the CCD is not illuminated and is almost constant in time which makes subtraction possible. The smear effect originates from the camera's frame transfer operation and is caused by the $310 \mu \mathrm{s}$ long time interval needed to shift the photoelectrons to the non-illuminated part of the CCD. During this shift-time, the pixels in a given row gather light from the other channels which results in a smeared contribution. When using short exposure times, such that the shift time is a significant fraction of the integration time, this spectral component must be removed. Therefore, a 16th, non-illuminated camera-channel has been defined that only measures the smeared light, which can subsequently be subtracted from the illuminated channels. Uncertainties in the intensity calibration on the order of $10 \%$ can arise due to damage or coating of the in-vessel optics during an experimental campaign. In addition, changing the fibers on the switch panel can yield uncertainties because the fiber connections are not $100 \%$ reproducible, damage to the fiber optics can occur, and the transmission properties can change (e.g. due to dust getting between the connection of two fibers). Therefore, the amount of switching is kept as minimal as possible and any relative changes that are observed are corrected by analyzing the level of Bremsstrahlung in the spectra, which should monotonically increase from the plasma edge to the plasma center. 


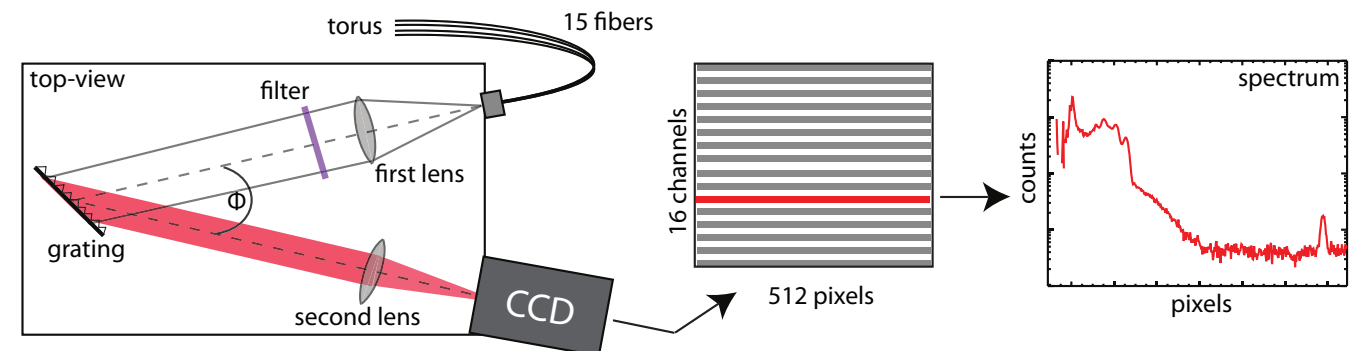

FIG. 3. Schematic top-down view on the FIDA spectrometer and camera setup.
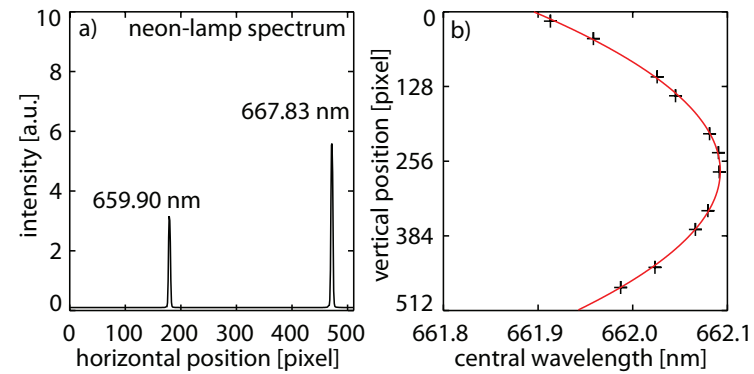

FIG. 4. a) Measured spectrum of the neon lamp between $655 \mathrm{~nm}$ and $668 \mathrm{~nm} \mathrm{~b}$ ) Central wavelength on the CCD as a function of the channel's vertical position on the chip. A fit to the data with a parabola is shown in red.

\section{PASSIVE RADIATION IN FIDA SPECTRA}

The spectra measured with the FIDA diagnostic contain active and passive radiation. The active radiation, such as the FIDA radiation discussed later, is only present during the operation of the diagnostic beam (here NBI Q3). In contrast, the passive radiation does not depend on NBI heating and is always present during plasma operation. Example spectra that were measured in discharge \#27679 without NBI heating are shown in figure $5 \mathrm{a}$ and $\mathrm{b}$ for a toroidal and a poloidal LOS, respectively. Only wavelengths above $655 \mathrm{~nm}$, i.e. red-shifted wavelengths, are monitored as the interference filter, described above, was used in the spectrometer. The data plotted in black was acquired in the presence of an edge-instability, i.e. an ELM-crash ${ }^{21}$, and the spectra in gray correspond to a quiescent plasma phase. The spectra during the ELM crash clearly exhibit an increased level of the individual passive contributions which will be described in the following subsections.

\section{A. Bremsstrahlung}

The flat background radiation, indicated by green dashed lines in figure 5, is due to the bremsstrahlung. Bremsstrahlung is mainly caused by electrons that are deflected via coulomb collisions and is emitted in the whole plasma. Its level in the spectra scales with

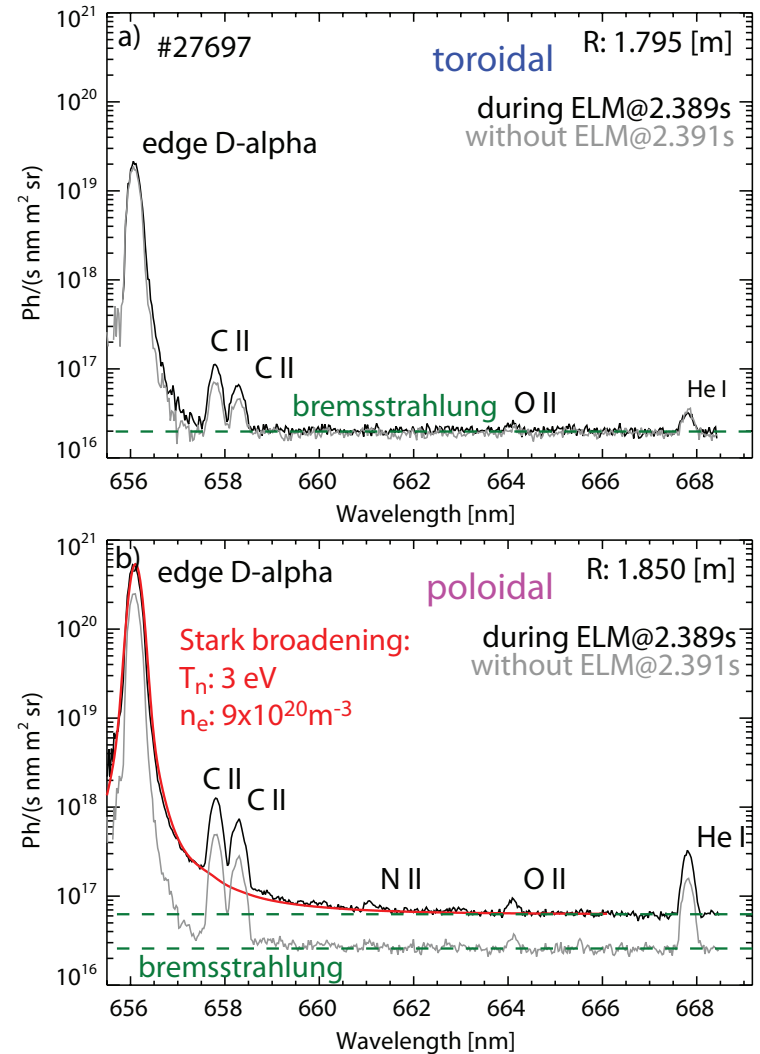

FIG. 5. Passive spectra from a toroidal LOS (a) and a poloidal LOS (b) that were observed during (black) and after (gray) an ELM crash. In red, the theoretical shape of the D-alpha emission is plotted, demonstrating the presence of Stark broadening of this line.

$\frac{n_{e}^{2} Z_{\text {eff }}}{\sqrt{T_{e}}}$, where $n_{e}$ is the electron density, $Z_{\text {eff }}$ the effective charge and $T_{e}$ the electron temperature that are encountered along the path of a given LOS. The level of bremsstrahlung measured by the poloidal LOS during the ELM crash is significantly increased compared to the quiescent time period because ELM crashes cause high densities and sputtered impurities in the divertor region. The poloidal LOS, as shown in figure 1, end in the vicinity of this region and, hence, can measure large levels of bremsstrahlung.

Due to its flat spectral shape, the bremsstrahlung can 
be modeled via a constant line. The height of this line can be measured at spectral positions that are not populated by line emissions (e.g. between $665 \mathrm{~nm}$ and $667 \mathrm{~nm}$ ). However, even though the bremsstrahlung can be accounted for by this method, high levels can significantly increase the photon noise in the spectra which can limit the analysis of the FIDA radiation. During ELMs, for example, the poloidal measurements are typically corrupted and the corresponding time intervals must be sorted out.

\section{B. Edge-D-alpha radiation}

In addition to the bremsstrahlung, the edge D-alpha emission line at $656.1 \mathrm{~nm}$ can be problematic for the measurements during ELM crashes. This component is one of the brightest line emissions of D-plasmas in the visible range. It is caused by electron impact excitation and by charge exchange reactions of deuterium neutrals and ions at the plasma edge and can routinely be monitored thanks to the interference filter in the spectrometer. The filter only attenuates the edge D-alpha line. It does not completely block it and its transmission properties can be taken into account in the diagnostic's intensity calibration. As can be seen in figure 5, the edge D-alpha radiation from the poloidal LOS is about 100 times more intense than that measured by the toroidal LOS. It has a non-Gaussian shape with a clear wing towards larger wavelengths. The origin of this wing is most likely due to Stark broadening which is caused by electric micro fields created by the high density of nearby charged particles. The more charge particles present, the larger the local electric fields, and the stronger the effective Stark splitting/broadening. The red line in figure $5 \mathrm{~b}$ shows the tabulated ${ }^{22}$ theoretical shape of the D-alpha line due to Stark and Doppler broadening, based on the model micro-field method $(\mathrm{MMM})^{23}$. The predicted spectral shape has been convoluted with the diagnostic's instrument function and corresponds to a neutral temperature of $3 \mathrm{eV}$ which is close to the Franck Condon dissociation energy of recycled neutrals. The electron density needed to obtain good agreement between the measured and theoretical shape is $9 \times 10^{20} \mathrm{~m}^{-3}$, which is relatively high compared to the values in the plasma center. However, electron densities of the order of $10^{21} \mathrm{~m}^{-3}$ are observed in the divertor region during ELM crashes ${ }^{24}$. The wing of the edge D-alpha line, measured by the poloidal LOS, reaches well above $659 \mathrm{~nm}$. As will be discussed later, the FIDA radiation is analyzed in this wavelength range and the effect of Stark broadening could falsely be identified as a fast-ion contribution. Therefore, measurements in the presence of high electron densities must be avoided. However, it should be noted that the spectra from the toroidal and the MSE LOS are almost unaffected by high densities in the divertor region. This permits unperturbed measurements with these LOS also during edge-instabilities.

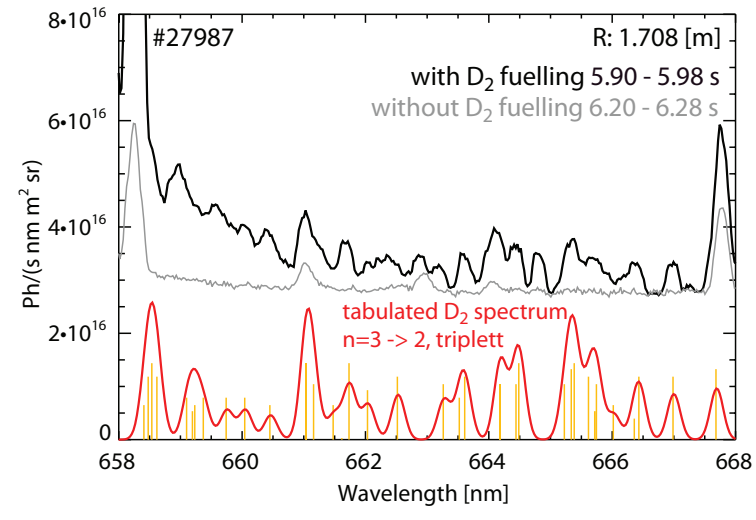

FIG. 6. $\mathrm{D}_{2}$ Spectra from a toroidal LOS, measured with (black) and without (gray) $\mathrm{D}_{2}$ fueling from a proximate valve. In orange, tabulated $\mathrm{D}_{2}$ molecular lines ${ }^{25}$ are shown that correspond to $n=3 \rightarrow 2$ transitions of the $\mathrm{D}_{2}$ triplet system.

\section{Impurity line emissions}

As indicated in figure 5, several impurity line emissions from the plasma edge are visible in the spectra of the FIDA diagnostic. The $C I I$ doublet at about $658 \mathrm{~nm}$ and the $\mathrm{He} I$ line emission at $667.8 \mathrm{~nm}$ are routinely observed with significant intensities. In addition, line emissions such as a $N I I$ line at $661.3 \mathrm{~nm}$ and an $O I I$ line at $664.1 \mathrm{~nm}$ can appear depending on the impurity content of the plasma. Compared to other machines like DIII-D ${ }^{13}$, TEXTOR ${ }^{12}$ or MAST ${ }^{14}$, the level of passive impurity line emissions in ASDEX Upgrade plasmas is very low, which significantly facilitates the analysis of the FIDA radiation. This is in part due to the fully tungsten coated walls that yield a strongly reduced content of carbon and in part due to frequently applied boronizations that cover the walls with a thin layer of boron. Boron absorbs oxygen chemically and consequently decreases the impurity content. Boron itself does not emit radiation between $659 \mathrm{~nm}$ and $669 \mathrm{~nm}$.

\section{D2 molecular lines}

$\mathrm{D}_{2}$ molecular lines represent another possible contribution in the spectra (not visible figure 5). They can be observed with the FIDA diagnostic during $\mathrm{D}_{2}$ fueling from valves situated close to the LOS and exist over a wide range of wavelengths: The molecular lines correspond to transitions between various electronically excited levels. Due to the presence of vibrational and rotational sublevels in these electronic states, the emission spectrum shows a distinct vibro-rotational structure. Figure 6 shows two spectra from a toroidal LOS that were measured during (black) and in the absence (gray) of $\mathrm{D}_{2}$ fueling. Clearly, a significant increase of the passive radiation is measured when $\mathrm{D}_{2}$ is injected through valves located in the vicinity of the LOS. In orange, tabulated line intensities ${ }^{25}$ of the $n=3 \rightarrow 2$ transitions of the 


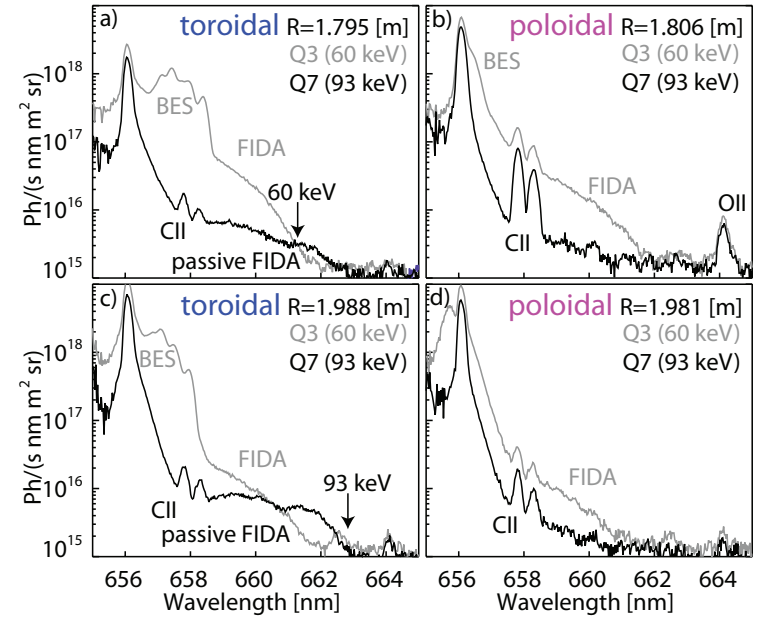

FIG. 7. Passive spectra from two toroidal LOS (a, c) and two poloidal LOS (b, d) in black that were acquired during off-axis NBI heating. In gray, active spectra are plotted that were acquired during the operation of NBI Q3.

$\mathrm{D}_{2}$ triplet system are plotted where $n$ denotes the main quantum number of the electronic states in the united atom approximation ${ }^{26}$. The corresponding theoretical spectrum that represents the sum of the single transitions, convoluted with the FIDA diagnostic's instrument function, is shown in red. Its shape agrees well with the measurement and hence evidences the existence of the molecular lines. Since the $\mathrm{D}_{2}$ lines are present across the whole spectrum, they would consequently be superimposed on the FIDA radiation. When performing dedicated fast-ion measurements it is therefore necessary to apply $\mathrm{D}_{2}$ fueling from valves far from the diagnostic's LOS. This guarantees almost $\mathrm{D}_{2}$ line-free spectra.

\section{E. Passive FIDA radiation}

As already explained in the introduction, the FIDA radiation is emitted by fast deuterium ions after charge exchange reactions with neutrals. In particular, along NBI lines strong, core localized, active FIDA signals can be observed because NBI provides a neutral population in the plasma core with which the fast ions can charge exchange. Edge localized, passive FIDA radiation can, in contrast, only be observed when a significant part of the fast-ion population undergoes charge exchange reactions with neutrals from the plasma periphery. The passive FIDA radiation can therefore mainly be observed during off-axis NBI heating because this generates large off-axis fast-ion populations. The strongest passive FIDA signals, yet observed, are visible in the black spectra in figure 7. The spectra were measured in discharge \#28881 during off-axis NBI heating from a $93 \mathrm{keV}$ source, labeled Q7. The measurement during off-axis NBI was clearly of passive nature since there is no overlap between the FIDA diagnostic's LOS and the path of Q7. As sketched in figure 1, NBI source Q7 belongs to box 2 which is displaced in the toroidal direction by $180^{\circ}$, relative to box 1. The passive spectra are shown for two toroidal and two poloidal LOS that intersect NBI Q3 at different radial positions. A significant spectral contribution between $658 \mathrm{~nm}$ and $663 \mathrm{~nm}$ can be observed with maximum wavelength shifts that correspond to the NBI injection energy of $93 \mathrm{keV}$. This passive FIDA component can be seen best in the toroidal spectrum in figure $7 \mathrm{c}$. The corresponding LOS is tangential to the edge-region and efficiently collects radiation from the passive FIDA emission layer. The passive radiation in the poloidal spectra (figures $7 \mathrm{~b}$ and d) is significantly weaker than that in the toroidal spectra (figures $7 \mathrm{a}$ and $\mathrm{c}$ ). First, the toroidal LOS are more sensitive to the passing fast ions generated by NBI Q7 (discussed in the following). Second, the fast-ion orbits are displaced relative to the flux surfaces radially outwards. Thereby they encounter large edge neutral densities close to the mid-plane. While the toroidal lines of sight intersect this region, the poloidal LOS do not.

As shown in gray in figure 7 , the level of active radiation measured during the operation of NBI Q3 only is not significantly higher than that of the passive signal. In FIDA measurements during off-axis NBI heating, the passive contribution must consequently be taken into account. As modelling of the passive FIDA component is difficult (the edge neutral density is not well known) the passive FIDA light must be measured in phases when the diagnostic beam NBI Q3 is off. It can then, if necessary, be subtracted from the active signals.

\section{ACTIVE RADIATION IN FIDA SPECTRA}

The active radiation is only present in FIDA spectra at ASDEX Upgrade during the operation of NBI Q3. Theoretically, it consists of three D-alpha components, i.e. the beam emission, the halo emission and the FIDA emission, and of charge exchange radiation from impurities. The active line emissions from impurities are, however, not observed under standard experimental conditions. Only during plasmas in a poorly conditioned machine, a CVI $(15-11) \operatorname{line}^{27}$ at $662.4 \mathrm{~nm}$ can be seen. In addition, an $\operatorname{ArXVI}(16-15)$ line appears in the spectrum at $661.5 \mathrm{~nm}$ during dedicated argon seeding experiments.

The three active D-alpha components are consequently the main active contributions. Their spectral shapes and origins will be discussed in this section using example spectra from a toroidal, a poloidal and the MSE LOS. The example spectra, as plotted in figure 9, are from a discharge that was heated by $5 \mathrm{MW}$ of NBI power and featured a very low electron density (see figure 8). This discharge was selected, not because the FIDA measurement exhibits particularly good signal to noise ratio (it is actually rather poor, e.g. compared to figure 7), but because it was possible to analyze the red- and blue-shifted sides of the spectrum. Due to the low plasma density, 


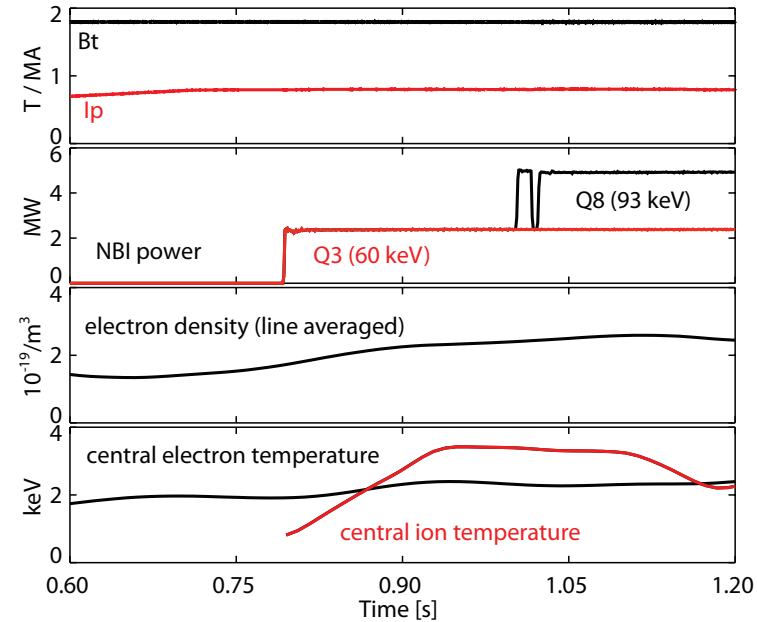

FIG. 8. Characteristic time-traces of discharge \#29578. From top to bottom, the plasma current and magnetic field, the NBI heating power, the electron density and the ion and electron temperatures are plotted.

the diagnostic could be operated without interference filter and without saturation effects from the edge D-alpha line. In addition to the experimental data, figure 9 shows theoretical spectra of the beam, halo and FIDA radiation from FIDASIM ${ }^{28}$ which are plotted on top of the level of the bremsstrahlung, visible above $663 \mathrm{~nm}$. FIDASIM is a Monte Carlo code that predicts the FIDA radiation that would be collected by an arbitrary LOS in the presence of a given theoretical fast-ion distribution function. The simulation uses atomic data, kinetic plasma profiles, the LOS geometry and the energy, power and geometry of the diagnostic beam. Additionally, it models the radiation from beam and halo neutrals. These two populations are needed to calculate the charge exchange probability of fast ions and, hence, are also simulated. As can be seen in figure 9 , the simulated components, which will be described in detail in the following, exhibit very good agreement with the measurement.

\section{A. Beam emission}

The simulated beam emission spectrum (BES) is displayed in figure 9 in orange/yellow. It originates from the injected deuterium neutrals that get excited as they move through the plasma and then emit D-alpha radiation. The beam emission only populates a well-defined part of the spectrum because the NBI generates neutrals with a narrowly focused velocity distribution that depends on the NBI geometry. Despite the high velocity of the injected neutrals, the observed wavelength shifts are relatively small, because the FIDA diagnostic's LOS intersect NBI Q3 almost perpendicularly. The toroidal LOS intersect NBI Q3 with angles of about $75^{\circ}$ and measure red-shifted beam emission. The poloidal LOS have angles to the NBI path between $80^{\circ}$ in the center and
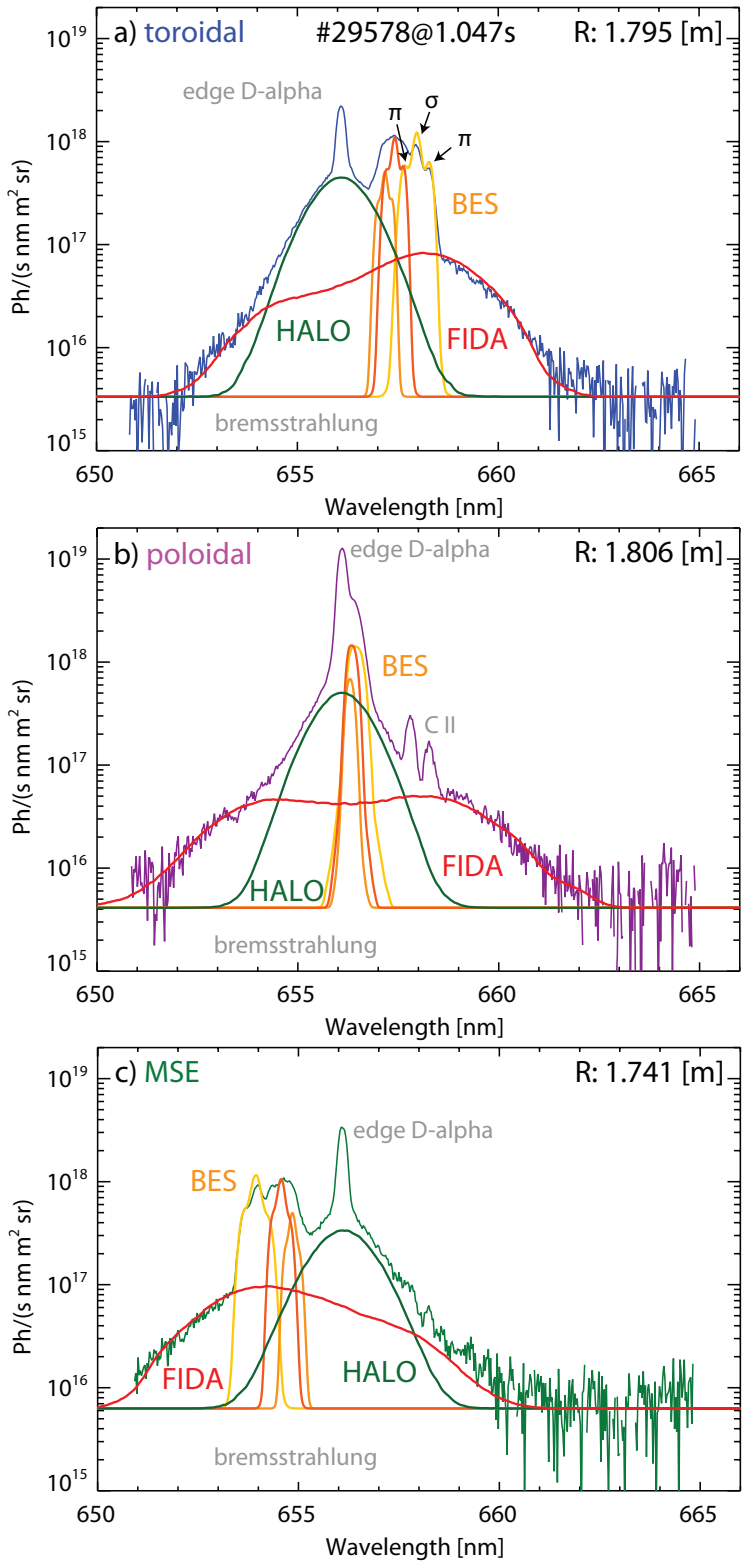

FIG. 9. Measured spectra on a semi-logarithmic scale from a toroidal LOS (a), a poloidal LOS (b) and the MSE LOS (c). In addition, the theoretical beam, halo and FIDA components predicted by FIDASIM are plotted.

$110^{\circ}$ degree close to the plasma edge and measure the beam emission from slightly red-shifted to slightly blueshifted, respectively. The MSE LOS points anti-parallel to the NBI path and, therefore, observes blue shifted beam emission.

The spectral shape of the beam emission depends on the NBI source's species mix, i.e. the injection of neutrals with the full, half and one third energy (shown with colors from yellow to orange) and on the Stark effect. The latter is imposed by the strong $\mathbf{v} \times \mathbf{B}$ electric fields experienced by the neutrals and causes a splitting of each energy component into roughly one un-shifted $\sigma$ (perpen- 
dicular to the electric field) and two shifted $\pi$ (parallel to the electric field) lines, as indicated in figure 9a for the full energy component. In addition to the theoretical ratio of the intensities of the sigma $\sigma$ and $\pi$ lines, the optical components of the diagnostic transmit this polarized light with different intensities. The toroidal LOS of the FIDA diagnostic, for example, do not transmit the $\sigma$ and $\pi$ components equally but with a ratio of $\approx 0.9$ due to the use of a mirror in the light path.

The absolute intensity of the beam emission and its radial dependence are mainly linked to plasma density. The higher the density is, the more injected neutrals get excited when entering the plasma and the higher the levels of the beam emission measured by LOS close to the plasma edge are. In the plasma center, in contrast, higher plasma densities yield reduced levels of the beam emission as the injected neutrals are more efficiently attenuated and fewer reach the plasma core. Here, it should be noted that the measurement of the beam emission is very important for the proper analysis of the FIDA radiation. When comparing the measured FIDA signals to modelling results from FIDASIM, the beam emission can be used to validate e.g. the electron density profiles used in the simulation. The density of injected beam neutrals, and hence also the simulated FIDA component can only be trusted if the simulated beam emission agrees with the measurement.

\section{B. Halo emission}

In addition to the beam-emission, also the halo emission should be used to validate the modelling results. The halo emission, as plotted in green in figure 9, is emitted by a cloud of thermal deuterium neutrals that is present along the NBI path and which contributes, in large part, to the charge exchange signal from fast ions. The so-called halo neutrals themselves also originate from charge exchange reactions between thermal deuterium ions and the injected beam neutrals, as well as with other halo neutrals. The spectral shape of the halo component can be approximated by a Gaussian curve whose width and position are linked to the ion temperature and rotation. Depending on these two quantities, the wings of the halo component can extend to relatively large wavelength shifts in the spectra. However, for wavelengths above $659 \mathrm{~nm}$ and below $653 \mathrm{~nm}$, the halo radiation is negligible under standard conditions, such as temperatures below $6 \mathrm{keV}$ and plasma rotations below $250 \mathrm{~km} / \mathrm{s}$. The intensity of the halo emission is linked to the beam neutral density but also depends on the impurity content of the plasma. The higher the impurity content, the lower the deuterium density. Hence there are fewer Dions present that can be neutralized and fewer thermal neutrals emit D-alpha light.

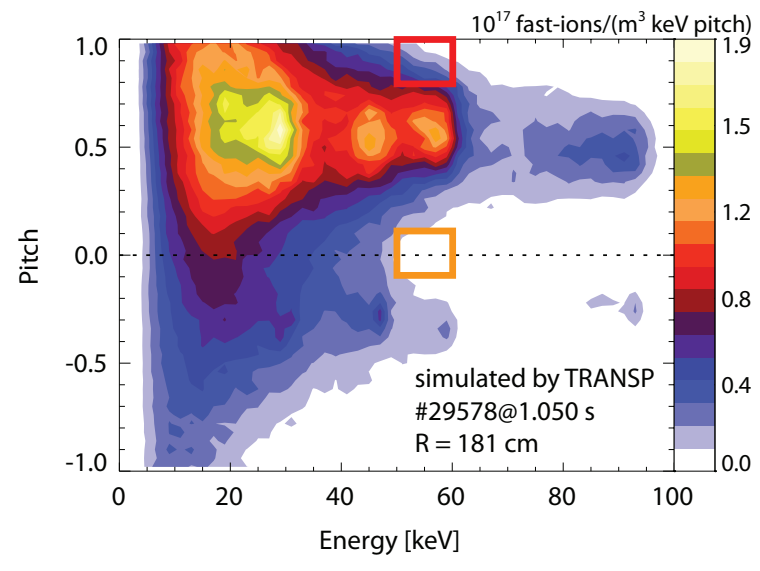

FIG. 10. TRANSP predicted velocity space distribution of fast ions in ASDEX Upgrade generated by NBI Q3 and a second $93 \mathrm{keV}$ heating beam in discharge \#29578 at $1.05 \mathrm{~s}$.

\section{FIDA emission}

The red curves in figure 9 show the simulated FIDA radiation which is based on a TRANSP ${ }^{29}$ predicted slowing down distribution function of fast ions. The corresponding velocity space distribution at about $R=1.81 \mathrm{~m}$ is plotted in figure 10 as a function of energy and pitch where pitch $=\mathrm{v}_{\|} / \mathrm{v}$, with $\mathrm{v}_{\|}$the fast-ion velocity antiparallel to the magnetic field lines, pointing into the cocurrent direction (see figure 1). The displayed velocity distribution is a good representation for ASDEX Upgrade since all of the eight available NBI sources are tilted into the co-current direction and hence primarily generate co-rotating fast ions. The beam-ion distribution functions in ASDEX Upgrade are consequently asymmetric in the pitch direction and have maximums close to 0.5 . The plotted velocity space exhibits energies up to $93 \mathrm{keV}$ because discharge \#29578 was heated by a $93 \mathrm{keV}$ NBI source in addition to the $60 \mathrm{keV}$ NBI source Q3. Due to these relatively large energies, in combination with a broadening of the distribution function in the pitch range due to collisions and different fast-ion deposition locations, the FIDA radiation can be observed at wavelengths above the beam and halo emissions. In figure 9, the measured and simulated FIDA components are clearly not overlaid below $653 \mathrm{~nm}$ and above $659 \mathrm{~nm}$. This makes the analysis of fast ions in these regions possible. The parts of the fast-ion velocity space that are observed at these wavelengths depend on the viewing geometries. As an example, figure 11 shows synthetic FIDA spectra of two different artificial velocity space distributions for the three viewing geometries. The velocity distribution used to calculate the spectra in figure 11a is highlighted by a red box in figure 10 . It consists of fastion energies between $50 \mathrm{keV}$ and $60 \mathrm{keV}$ and of pitches between 0.8 and 1.0 and hence represents passing fast ions that move in the co-current direction. Clearly, only the toroidal LOS and the MSE LOS are able to measure 

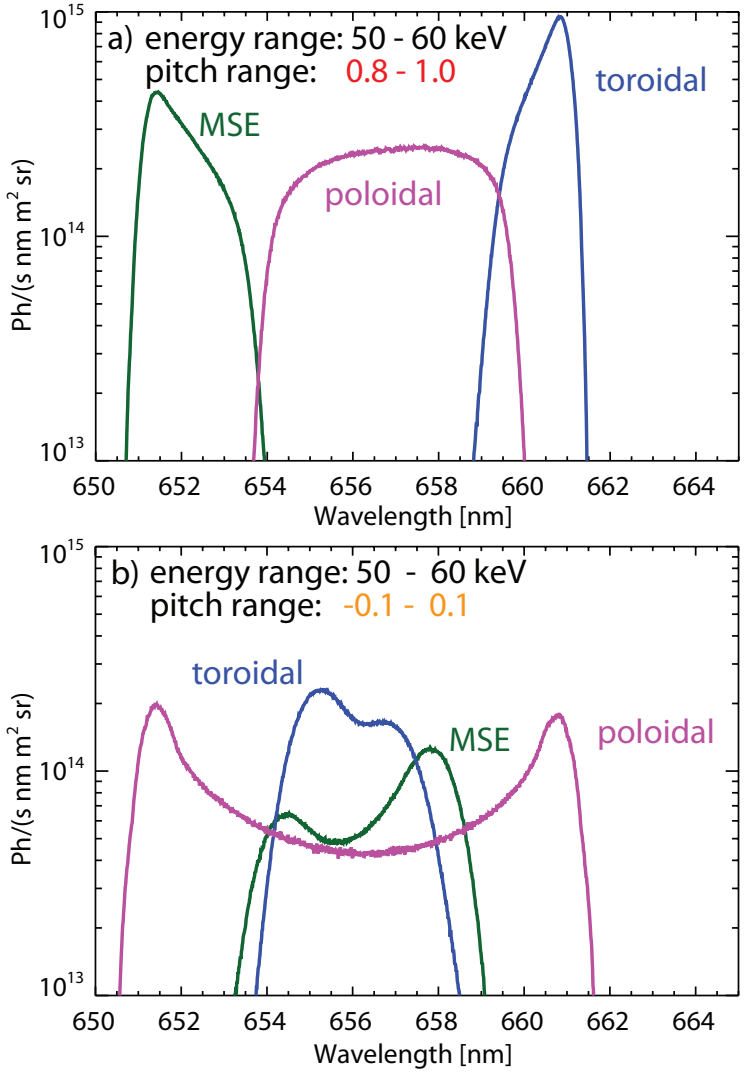

FIG. 11. Synthetic FIDA spectra observed by a toroidal (blue), poloidal (purple) and the MSE (green) LOS for the two artificial fast-ion distributions sketched in figure 10 in red and orange. a) Spectra for pitches between 0.8 and 1.0. b) Spectra for pitches between -0.1 and 0.1 .

these ions well at wavelengths below $653 \mathrm{~nm}$ and above $659 \mathrm{~nm}$ because such Doppler shifts are only observed when fast ions move parallel or anti-parallel to the LOS. Since the LOS have well defined angles to the magnetic field lines, parallel moving fast ions have explicit pitch values as well. Fast-ions that propagate parallel to the toroidal LOS have pitches of $\cos \left(12^{\circ}\right)=0.98$. Those propagating anti-parallel to the MSE LOS have pitches of $\cos \left(180^{\circ}-157^{\circ}\right)=0.92$. In the toroidal spectra, the simulated fast ions with pitches between 0.8 and 1.0 consequently cause strongly red-shifted FIDA radiation. In the MSE spectrum, strongly blue shifted radiation is observed from these ions. When, in contrast simulating spectra for a fast-ion velocity distribution with pitch values between -0.1 and 0.1 (orange box in figure 10) only the poloidal spectrum exhibits wavelengths below $653 \mathrm{~nm}$ and above $659 \mathrm{~nm}$, as shown in figure 11b. Fast ions with pitches close to zero move almost parallel or anti-parallel to the poloidal LOS and hence cause large Doppler shifts (the largest Doppler shifts would be expected from fast ions with pitch values of $\left.\cos \left(70^{\circ}\right)=0.34\right)$.

An alternative method to illustrate the dependence between the measured wavelength shifts and the observed parts of the fast-ion velocity space is provided by so called

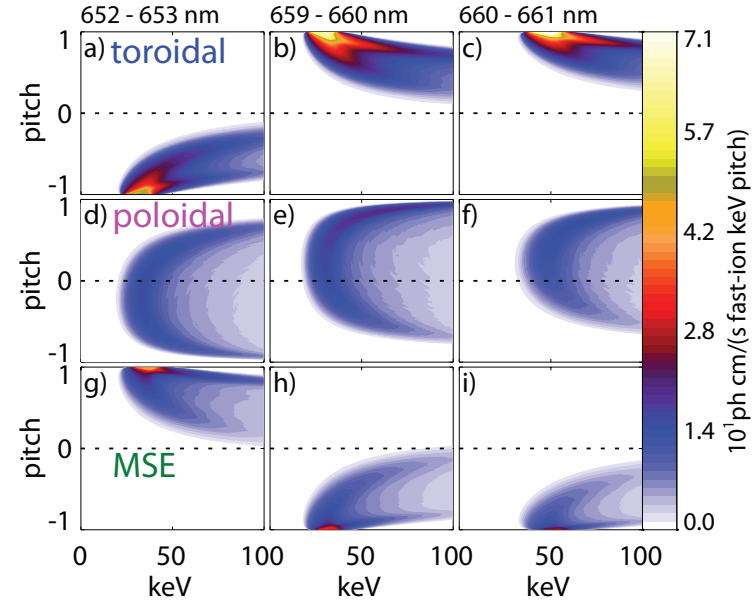

FIG. 12. Weight functions for a toroidal, poloidal and the MSE LOS for three different wavelength ranges. Depending on the wavelength, the LOS access different parts of the fastion velocity space.

diagnostic weight functions. A grid of example weight functions for three LOS and for three wavelength intervals is shown in figure 12. For a defined wavelength interval and LOS, the weight functions provide the number of photons that would be detected from a single fast-ion in a given pitch and energy range. They account for the charge exchange probability of the fast ions, for the photon emission probability and for the Doppler shift and Stark splitting. According to the weight functions shown in figures $12 \mathrm{~d}$, e and $\mathrm{f}$, the poloidal LOS are sensitive to fast ions with pitch values close to zero, i.e. trapped fast ions. They cover a wide range of pitches for blue-shifted (figure 12d) and red-shifted (figures 12e and $\mathrm{f}$ ) wavelengths. The toroidal and the MSE LOS, in contrast, cover more narrow ranges in the pitch space. They show a roughly symmetric behavior in pitch with opposite signs. At the blue shifted side of the spectrum, the toroidal LOS is sensitive to counter-rotating fast ions while the MSE LOS measures co-rotating fast ions and vice versa for the red-shifted side.

To illustrate the parts of the velocity space that can actually be investigated in NBI-heated plasmas in ASDEX Upgrade, figure 13 shows the product of the weight functions and the representative fast-ion velocity distribution, displayed in figure 10. This product yields the number of photons/(s sr nm $\left.\mathrm{m}^{2}\right)$ that would be observed by a given LOS in a set wavelength range as a function of energy and pitch. With the individual LOS and wavelength ranges, distinct parts of the velocity space are clearly measured. This allows, for example, the effect of sawtooth crashes ${ }^{30}$ on the different parts of the velocity space, to be investigated. In addition, the acquisition of FIDA spectra with multiple viewing geometries, as presented here, will open up the possibility to infer $2 \mathrm{D}$ velocity-space distribution functions from the spectra by tomographic inversion in velocity space as presented in ${ }^{31,32}$. 


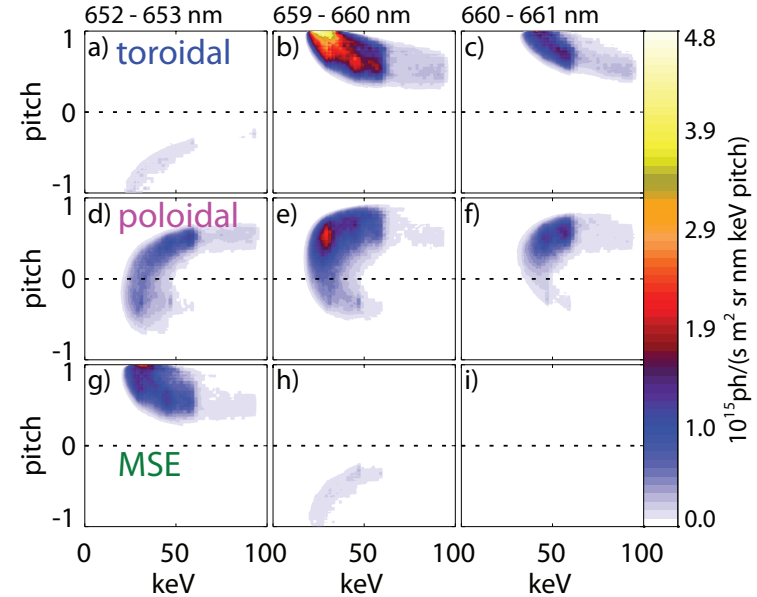

FIG. 13. Product of the fast-ion velocity distribution shown in figure 10 with the weight functions displayed in figure 12 .
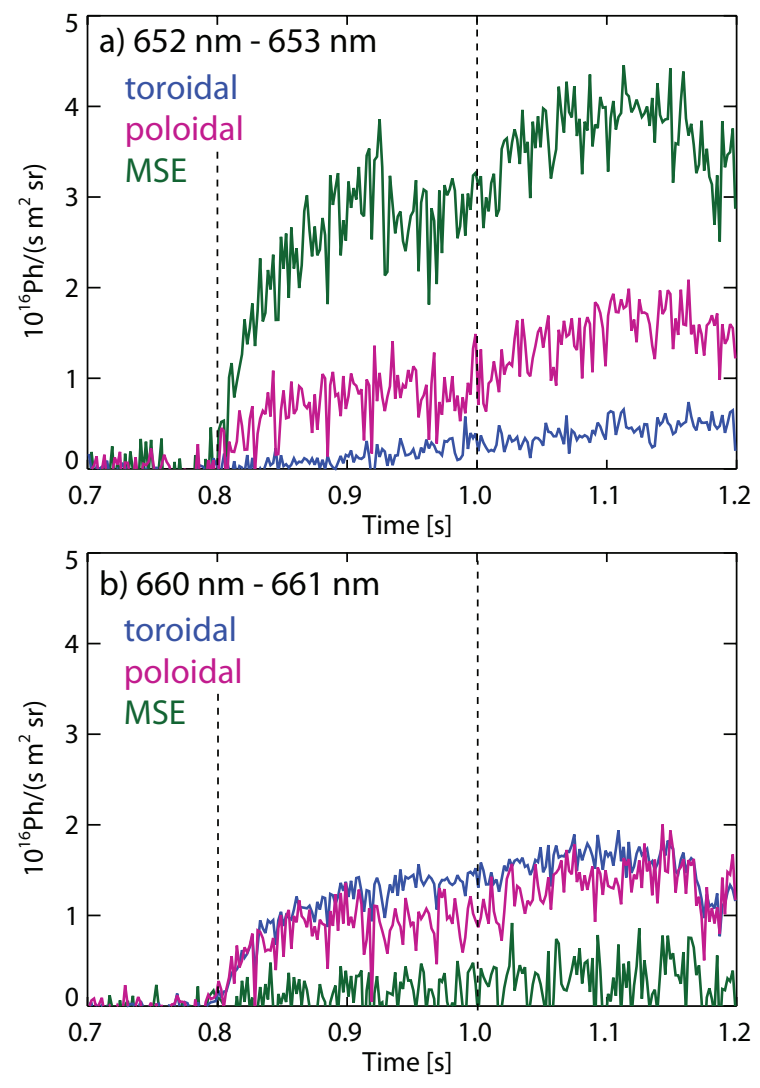

FIG. 14. Temporal evolution of the FIDA intensities for three LOS and two wavelength ranges $(a, b)$.

\section{Time traces and radial profiles of the FIDA light}

The FIDA diagnostic's exposure time of $2 \mathrm{~ms}$ permits the analysis of the evolution of measured FIDA intensities with good temporal resolution. FIDA intensities measured by each LOS can be calculated by integrating the spectra in wavelength and by modelling the background

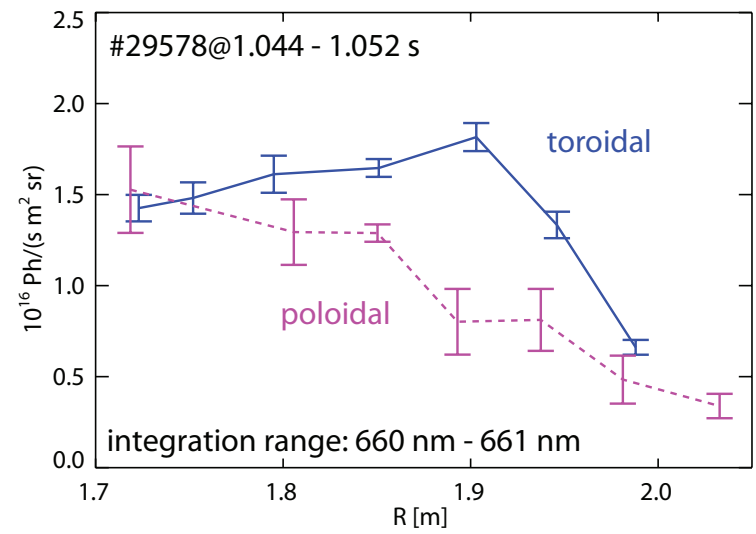

FIG. 15. Radial FIDA intensity profiles from the 7 toroidal LOS (blue) and 7 poloidal (purple) LOS that were connected to the FIDA spectrometer during discharge \#29578. The error-bars correspond to the fluctuation of the FIDA signal in the analyzed, $8 \mathrm{~ms}$ long, time interval.

radiation with a flat line. The height of this flat line, representing the bremsstrahlung, can be derived from parts of the spectra which are free from line radiation. The FIDA intensities depend on the fast-ion distribution and on the density of beam and halo neutrals along the path of NBI Q3. If this density can be assumed to be constant in time, the changes of the FIDA intensity can already indicate changes of the fast-ion confinement. Figures 14a and $b$ show FIDA intensities from three LOS and for two different wavelength integration ranges, measured in discharge \#29578. The FIDA radiation appears after NBI Q3 is turned on at about $0.8 \mathrm{~s}$ and increases further at $1.0 \mathrm{~s}$ when the second NBI source, Q8, is added. The data in figure 14 a correspond to the wavelength range between $652 \mathrm{~nm}$ and $653 \mathrm{~nm}$ and hence represent the regions of the velocity space displayed in figures $12 \mathrm{a}, \mathrm{d}$ and g. While the MSE LOS measures strong levels of the FIDA radiation, the poloidal LOS measures weak levels and the toroidal almost nothing. This is well explained by figures $13 \mathrm{a}, \mathrm{d}$ and $\mathrm{g}$, which show the overlap of the corresponding weight function with the fast-ion velocity space. However, it should be noted that the velocity space distribution used to calculate the overlap in figure 13 only corresponds to one time point at $1.05 \mathrm{~s}$ in the analyzed discharge. It, for example, does not exactly describe the velocity space when only fast ions from the $60 \mathrm{keV}$ source are present and, hence, only gives a rough estimate. The time traces of the FIDA signal in figure $14 \mathrm{~b}$ correspond to fast ions with energies above $30 \mathrm{keV}$ because an integration range between $660 \mathrm{~nm}$ and $661 \mathrm{~nm}$ has been used. In this integration range, the toroidal and poloidal LOS measure similar intensities while the velocity space observed by the MSE LOS clearly has no significant overlap with the fast-ion distribution function.

In addition to the analysis of time traces, the FIDA intensities per LOS can be used to analyze radial profiles. As an example, figure 15 shows radial profiles from 
the toroidal LOS (solid line) and from the poloidal LOS (dashed line) that represent the FIDA radiation between $660 \mathrm{~nm}$ and $661 \mathrm{~nm}$. The profiles exhibit different spectral shapes as different regions in the velocity space are observed. The error bars of the data represent two sigma of the scatter of the FIDA data which has been averaged over a time interval of $8 \mathrm{~ms}$. In future experiments, the analysis of radial profiles will provide, for example, better insight into the current drive capabilities of the off-axis NBI sources ${ }^{33}$.

\section{SUMMARY AND OUTLOOK}

A new FIDA spectroscopy diagnostic has been installed at the ASDEX Upgrade tokamak that uses lines of sight (LOS) with three different viewing geometries. The LOS intersect a 2.5 MW NBI heating source at different positions and allow measurements with good radial resolution. A high photon throughput spectrometer has been built that is combined with a low noise EM-CCD camera to simultaneously measure the light from 15 LOS. By inserting an interference filter in the spectrometer setup, the intense passive D-alpha radiation from the plasma edge can be attenuated but still measured. Thereby, e.g. wide spectral wings of the passive $\mathrm{D}$-alpha radiation at $656.1 \mathrm{~nm}$ could be identified as Stark broadening. This effect and increased levels of the bremsstrahlung were found to corrupt measurements from the poloidal LOS during edge-instabilities. Moreover, molecular lines of the triplet system of $\mathrm{D}_{2}$ have been found to contaminate the FIDA spectra when using $\mathrm{D}_{2}$ fueling valves close to the LOS and passive FIDA radiation is observed, in particular by toroidal LOS, during off-axis NBI heating. Despite the extreme cases, presented in this work, the spectra at ASDEX Upgrade are normally unperturbed and allow the continuous measurement of the FIDA radiation. Depending on the observed spectral range and viewing geometry, different regions of the fast-ion velocity space are accessible. Passing fast ions as well as trapped fast ions can be measured which allow studies of the fastion velocity space. Thereby, also time traces and radial profiles of the FIDA radiation can be analyzed.

In the future, installing additional viewing geometries with angles to the magnetic field lines close to $40^{\circ}$ and $80^{\circ}$ will permit the direct measurement of the fast-ion velocity space distribution using tomographic inversion procedures as proposed by ${ }^{31,32,34}$. Moreover, the FIDA spectrometer could be equipped with a narrow blocking wire close to the CCD chip. This could replace the interference filter and would routinely enable the simultaneous measurements of the blue and red-shifted FIDA radiation.

${ }^{1}$ A. Herrmann, O. Gruber. Chapter 1: ASDEX Upgrade - Introduction and Overview. Fusion Science and Technology, 44(3): 569-577, 2003. URL http://epubs.ans.org/?a=399.

${ }^{2}$ A. Fasoli, C. Gormenzano, H.L. Berk, B. Breizman, S. Briguglio, D.S. Darrow, N. Gorelenkov, W.W. Heidbrink, A. Jaun, S.V.
Konovalov, R. Nazikian, J.-M. Noterdaeme, S. Sharapov, K. Shinohara, D. Testa, K. Tobita, Y. Todo, G. Vlad, and F. Zonca. Chapter 5: Physics of energetic ions. Nuclear Fusion, 47(6):S264, 2007. URL http://stacks.iop.org/0029-5515/47/i=6/a=S05.

${ }^{3}$ M. Cecconello, S. Sangaroon, M. Turnyanskiy, S. Conroy, I. Wodniak, R.J. Akers, G. Ericsson, and the MAST Team. Observation of fast ion behaviour with a neutron emission profile monitor in MAST. Nuclear Fusion, 52(9):094015, 2012. URL http://stacks . iop.org/0029-5515/52/i=9/a=094015.

${ }^{4}$ M. Nocente, M. Garcia-Munoz, G. Gorini, M. Tardocchi, A. Weller, S. Akaslompolo, R. Bilato, V. Bobkov, C. Cazzaniga, B. Geiger, G. Grosso, A. Herrmann, V. Kiptily, M. Maraschek, R. McDermott, J.M. Noterdaeme, Y. Podoba, G. Tardini, and the ASDEX Upgrade Team. Gamma-ray spectroscopy measurements of confined fast ions on ASDEX Upgrade. Nuclear Fusion, 52(9):094021, 2012. URL http://stacks.iop.org/0029-5515/ $52 / i=9 / a=094021$.

${ }^{5}$ M. Salewski, F. Meo, M. Stejner, O. Asunta, H. Bindslev, V. Furtula, S.B. Korsholm, T. Kurki-Suonio, F. Leipold, F. Leuterer, P.K. Michelsen, D. Moseev, S.K. Nielsen, J. Stober, G. Tardini, D. Wagner, P. Woskov, and the ASDEX Upgrade team. Comparison of fast ion collective thomson scattering measurements at ASDEX Upgrade with numerical simulations. Nuclear Fusion, 50 (3):035012, 2010. URL http://stacks.iop.org/0029-5515/50/ $i=3 / a=035012$.

${ }^{6}$ M. Garcia-Munoz, H.-U. Fahrbach, H. Zohm, and the ASDEX Upgrade Team. Scintillator based detector for fast-ion losses induced by magnetohydrodynamic instabilities in the ASDEX Upgrade tokamak. Review of Scientific Instruments, 80(5):053503, 2009. URL http://link.aip.org/link/?RSI/80/053503/1.

${ }^{7} \mathrm{M}$ R Tournianski, R J Akers, P G Carolan, and D L Keeling. Anisotropic fast neutral particle spectra in the MAST spherical tokamak. Plasma Physics and Controlled Fusion, 47(5):671, 2005. URL http://stacks.iop.org/0741-3335/47/i=5/a=001.

${ }^{8}$ S.J. Zweben, R.V. Budny, D.S. Darrow, S.S. Medley, R. Nazikian, B.C. Stratton, E.J. Synakowski, and G. Taylor for the TFTR Group. Alpha particle physics experiments in the tokamak fusion test reactor. Nuclear Fusion, 40(1):91, 2000. URL http://stacks.iop.org/0029-5515/40/i=1/a=307.

${ }^{9} \mathrm{~W}$ W Heidbrink, K H Burrell, Y Luo, N A Pablant, and E Ruskov. Hydrogenic fast-ion diagnostic using Balmer-alpha light. Plasma Physics and Controlled Fusion, 46(12):1855, 2004. URL http://stacks. iop.org/0741-3335/46/i=12/a=005.

${ }^{10}$ Masaki Osakabe, Sadayoshi Murakami, Mikirou Yoshinuma, Katsumi Ida, Allan Whiteford, Motoshi Goto, Daiji Kato, Takako Kato, Kenichi Nagaoka, Tokihiko Tokuzawa, Yasuhiko Takeiri, and Osamu Kaneko. Fast ion charge exchange spectroscopy measurement using a radially injected neutral beam on the large helical device. Review of Scientific Instruments, 79 (10):10E519, 2008. URL http://link.aip.org/link/?RSI/79/ 10E519/1.

${ }^{11}$ W. W. Heidbrink, R. E. Bell, Y. Luo, and W. Solomon. Fastion D-alpha diagnostic for NSTX. Review of Scientific Instruments, 77(10):10F120, 2006. URL http://link.aip.org/link/ ?RSI/77/10F $120 / 1$

${ }^{12}$ E. Delabie, R. J. E. Jaspers, M. G. von Hellermann, S. K. Nielsen, and O. Marchuk. Charge exchange spectroscopy as a fast ion diagnostic on TEXTOR. Review of Scientific Instruments, 79 (10):10E522, 2008. URL http://link.aip.org/link/?RSI/79/ 10E522/1.

${ }^{13}$ Y. Luo, W. W. Heidbrink, K. H. Burrell, D. H. Kaplan, and P. Gohil. Measurement of the $\mathrm{D}_{\alpha}$ spectrum produced by fast ions in DIII-D. Review of Scientific Instruments, 78(3):033505, 2007. URL http://link.aip.org/link/?RSI/78/033505/1.

${ }^{14} \mathrm{C}$ A Michael, N Conway, B Crowley, O Jones, W W Heidbrink, S Pinches, E Braeken, R Akers, C Challis, M Turnyanskiy, A Patel, D Muir, R Gaffka, and S Bailey. Dual view FIDA measurements on MAST. Plasma Physics and Controlled Fusion, 55(9): 095007, 2013. URL http://stacks.iop.org/0741-3335/55/i= $9 / a=095007$. 
${ }^{15} \mathrm{M}$ A Van Zeeland, W W Heidbrink, and J H Yu. Fast ion $\mathrm{D}_{\alpha}$ imaging in the DIII-D tokamak. Plasma Physics and Controlled Fusion, 51(5):055001, 2009. URL http://stacks.iop. org $/ 0741-3335 / 51 / i=5 / a=055001$.

${ }^{16}$ B Geiger, M Garcia-Munoz, W W Heidbrink, R M McDermott, G Tardini, R Dux, R Fischer, V Igochine, and the ASDEX Upgrade Team. Fast-ion D-alpha measurements at ASDEX Upgrade. Plasma Physics and Controlled Fusion, 53(6): 065010, 2011. URL http://stacks.iop.org/0741-3335/53/i= $6 / a=065010$.

${ }^{17}$ R. Reimer, A. Dinklage, J. Geiger, J. Hobirk, M. Reich, R. Wolf, ASDEX Upgrade, and Wendelstein 7-X Teams. Motional stark effect spectra simulations for Wendelstein 7-X. Contributions to Plasma Physics, 50(8):731-735, 2010. ISSN 1521-3986. URL http://dx.doi.org/10.1002/ctpp. 200900034.

${ }^{18}$ 2013. URL http://www.princetoninstruments.com/products/ imcam/proem/.

${ }^{19}$ A. Kramida, Yu. Ralchenko, J. Reader, and NIST ASD Team. NIST atomic spectra database (version 5.0). 2012. URL http: //physics.nist.gov/asd.

${ }^{20} 2013$. URL http://www. labsphere.com.

${ }^{21} \mathrm{H}$ Zohm. Edge localized modes (ELMs). Plasma Physics and Controlled Fusion, 38(2):105, 1996. URL http://stacks.iop. org $/ 0741-3335 / 38 / i=2 / a=001$.

${ }^{22}$ C. Stehlé and R. Hutcheon. Extensive tabulations of stark broadened hydrogen line profiles. Astron. Astrophys. SuppL. Ser., 140: 93-97, 1999.

${ }^{23}$ A. Brissaud and U. Frisch. Theory of Stark broadening II - exact line profile with Model Microfield. J. Quant. Spectrosc. Radiat. Transfer, 11:1767-1783, 1971.

${ }^{24}$ S. Potzel, M. Wischmeier, M. Bernert, R. Dux, H.W. Mller, and A. Scarabosio. Characterization of the fluctuating detachment state in ASDEX upgrade. Journal of Nuclear Materials, 438: S285 - S290, 2013

${ }^{25}$ R.S. Freund and J.A. Schiavone. Electronic spectrum and energy levels of the deuterium molecule. J. Phys. Chem. Ref., 14:235, 1985.

${ }^{26}$ Potential-energy curves for molecular hydrogen and its ions. Atomic Data and Nuclear Data Tables, 2(0):119 - 169, 1970. ISSN 0092-640X.

${ }^{27}$ J.D. GARCIA and J.E. MACE. Energy level and line tables for one-electron atomic spectra. J. Opt. Soc. Am., 55(6):654-661,
Jun 1965. URL http://www.opticsinfobase.org/abstract. cfm?URI $=$ josa $-55-6-654$.

${ }^{28}$ W. Heidbrink, D Liu, Y. Luo, E. Ruskov, and B. Geiger. A code that simulates fast-ion $\mathrm{D}_{\alpha}$ and neutral particle measurements. Commun. Comput. Phys., 10(10):716-741, 2011.

${ }^{29}$ Alexei Pankin, Douglas McCune, Robert Andre, Glenn Bateman, and Arnold Kritz. The tokamak monte carlo fast ion module NUBEAM in the national transport code collaboration library. Computer Physics Communications, 159(3):157 - 184, 2004.

${ }^{30}$ Ya.I. Kolesnichenko, V.V. Lutsenko, R.B. White, and Yu.V. Yakovenko. Effect of sawtooth oscillations on energetic ions. $\mathrm{Nu}$ clear Fusion, 40(7):1325, 2000. URL http://stacks.iop.org/ $0029-5515 / 40 / i=7 / a=304$.

${ }^{31}$ M. Salewski, B. Geiger, S.K. Nielsen, H. Bindslev, M. GarcaMuoz, W.W. Heidbrink, S.B. Korsholm, F. Leipold, F. Meo, P.K. Michelsen, D. Moseev, M. Stejner, G. Tardini, and the ASDEX Upgrade team. Tomography of fast-ion velocity-space distributions from synthetic CTS and FIDA measurements. $\mathrm{Nu}$ clear Fusion, 52(10):103008, 2012. URL http://stacks.iop. org/0029-5515/52/i=10/a=103008.

${ }^{32}$ M. Salewski, B. Geiger, S.K. Nielsen, H. Bindslev, M. GarcaMuoz, W.W. Heidbrink, S.B. Korsholm, F. Leipold, J. Madsen, F. Meo, P.K. Michelsen, D. Moseev, M. Stejner, G. Tardini, and the ASDEX Upgrade Team. Combination of fast-ion diagnostics in velocity-space tomographies. Nuclear Fusion, 53(6): 063019, 2013. URL http://stacks.iop.org/0029-5515/53/i= $6 / a=063019$

${ }^{33}$ S. Günter, G. Conway, S. daGraca, H.-U. Fahrbach, C. Forest, M. Garcia Munoz, T. Hauff, J. Hobirk, V. Igochine, F. Jenko, K. Lackner, P. Lauber, P. McCarthy, M. Maraschek, P. Martin, E. Poli, K. Sassenberg, E. Strumberger, G. Tardini, E. Wolfrum, H. Zohm, and ASDEX Upgrade Team. Interaction of energetic particles with large and small scale instabilities. Nuclear Fusion, 47(8):920, 2007. URL http://stacks.iop.org/0029-5515/47/ $i=8 / \mathrm{a}=025$.

${ }^{34}$ M. Salewski, B. Geiger, A.S. Jacobsen, M. Garcia-Munoz, W.W. Heidbrink, S.B. Korsholm, F. Leipold, J. Madsen, D. Moseev, S.K. Nielsen, J. Rasmussen, M. Stejner, G. Tardini, M. Weiland, and the ASDEX Upgrade team. Measurement of a 2D fast-ion velocity distribution function by tomographic inversion of fastion D-alpha spectra. in preparation, 2013. 